\title{
Design of the Airbag Inflation System Applicable to Conventional and Autonomous Vehicles
}

\author{
Nina F. Yurchenko ${ }^{1}$ (D) David S. Breed ${ }^{2} \cdot$ Shaowei Zhang ${ }^{3}$
}

Received: 22 October 2020 / Accepted: 3 June 2021 / Published online: 13 September 2021

(c) The Author(s) 2021

\begin{abstract}
The emergency transformation of various aspects of life and business these days requires prompt evaluation of autonomous vehicles. One of the primary reassessments deals with the applicability of the vehicle passive safety system to the protection of arbitrarily positioned passengers. To mitigate possible risks caused by the simultaneous deployment of several big airbags, a new principle of their operation is required. Herein, the aspirated inflator for a driver airbag is developed that can provide $50 L$-airbag inflation within $30-40 \mathrm{~ms}$. As a result, about $3 / 4$ of the air is to be entrained into an airbag from the vehicle compartment. The process is initiated by a supersonic pulse jet ( $1 / 3$ air volume) generated pyrotechnically. Then the Prandtl-Meyer problem formulation enables guiding linear and angular dimensions of the essential parts of the device. Accordingly, a family of experimental models of varied geometry is fabricated and tested to determine their operational effectiveness in a range of motive pressure within 3-7 MPa. Experiments are performed on a specially designed facility equipped with compressed-air tanks and a high-speed valve to mimic the inflator operation with the pyrotechnic gas generator. The aspirated inflator operability is characterized using multivariate measurements of pressure fields, high-speed videorecording of the airbag inflation process, and evaluation of aspiration (entrainment) ratio. The average volume aspiration ratio measured at $300 \mathrm{~K}$ is found to reach 2.8 and it's expected to almost double at $1200 \mathrm{~K}$.
\end{abstract}

Keywords Novel airbag system $\cdot$ Aspirated inflator $\cdot$ Air entrainment effectiveness $\cdot$ Experimental investigations

\section{Introduction}

The ongoing pandemic realities necessitate essential rearrangements of businesses and ordinary lifestyles. It reveals itself in the organization of remote operations, digitization, and robotization of various spheres of the human being. The development of new technologies and their integration with the market needs depend on the essential modernization of transportation services. To contribute to the rapid rehabilitation of industry and stable recovery of the population, safe

Nina F. Yurchenko

nina.yurchenko@gmail.com

1 Laboratory for Advanced Aerodynamics and Interdisciplinary Research, Institute of Hydromechanics, National Academy of Sciences of Ukraine, 8/4 Zheliabov St., Kyiv 03680, Ukraine

2 Automotive Technologies International, Inc. USA, 800 West Ave, Unit 545, Miami Beach, FL 33139, USA

3 Shanghai East Joylong Motor Airbag Co., Ltd., 219\# Qingda Road Pudong District, Shanghai, China and desirably contactless deliveries should be provided, especially in emergencies, where the least number of special personnel is to be involved.

Autonomous vehicles (AVs) significantly enhance the available transportation system. In the next two decades, mobility services will proliferate to meet the increasing travel demand [1]. For example, Tesla Motor launches the first robotaxis as part of a broader vision for an autonomous ride-sharing network. The FABULOS project (Future Automated Bus Urban Level Operation System) [2] is launched in 6 European urban areas (Fig. 1).

Fully automated vehicles and trucks that drive us, instead of us driving them, are becoming our everyday reality. According to the Global Times [3], China promotes its autonomous auto-driving industry, accelerating scientific and technological research and innovations. Besides changing the available automotive supply chain, AVs will require extensive systematic R\&D efforts to put in place protections for vehicle occupants.

Market forces encouraging the introduction of new technologies require legislation to speed the process. 


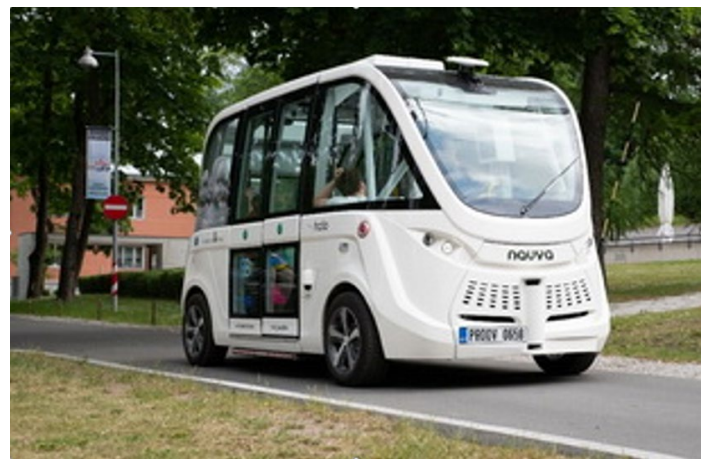

Fig. 1 Self-driving bus services tested out in Tallinn [2]

Aiming to reduce road casualties, policymakers are issuing new rules. The U.S. Department of Transportation's National Highway Traffic Safety Administration [4] removes regulatory barriers to innovative safety technology. A new European Union regulation calls for all new cars sold in Europe to be equipped with "advanced safety systems" starting from mid-2022 [5].

Automotive safety reveals new problems and importance for the stage of combined conventional and autonomous traffic. The software developed for autonomous vehicles to model drivers' human behavior will suffer from inevitable bugs to correctly predict actual human behavior. In other words, autonomous vehicles must deal in traffic with fallible human drivers that will endanger occupants of both cars. That is why the development of enhanced automotive safety systems is the primary task.

Nowadays, airbags together with seat belts are the primary safety systems in a car. Typically, the signals relayed from impact sensors activate propellant pellets to generate gas filling the cushion. Harmful effects include the high temperature of generated gases and their impact on the environment, rapidly rising internal pressure in the cushion and the cabin [6]. This impact is augmented in severe accidents and in autonomous vehicles where big airbags can be deployed simultaneously. It requires advanced engineering solutions to raise the safety of arbitrarily located vehicle occupants [7-10]. The aspirated inflation system can become such a solution [10-12], e.g., both for the German OEM ZF seatintegrated central airbag system Fig. 2a [7] and for the Autoliv versions of the airbag system for autonomous vehicles Fig. 2b, c [8] with arbitrarily located passengers.

Advantageous features of the aspirated inflators (Fig. 3) result from their mode of operation based on the air entrainment into an airbag from the car cabin [10-13]. They are as follows:

- Applicability to autonomous vehicles;

- Reduced amount of propellant (gas generator less than $1 / 3$ of a conventional size);

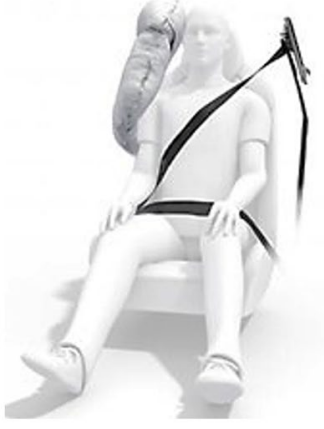

(a)

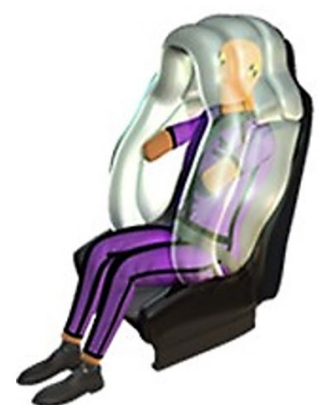

(b)

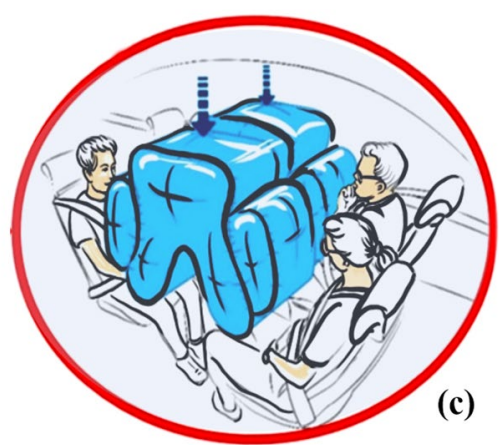

Fig. 2 Versions of airbags for autonomous vehicles: a ZF seat-integrated central airbag system; b life cell airbag, Autoliv; $\mathbf{c}$ operation of airbags with aspirated inflators in autonomous cars (Autoliv) in case of aspirated inflators used: reduced cabin pressure, no harm to occupants $[7,8]$

- Pressure and temperature control since most of the gas to inflate the airbag comes from the passenger compartment (Fig. 3);

- Stopped airbag deployment on contact with an occupant that eliminates the need for

- Out-of-position occupant sensors,

- Occupant weight and position sensors,

- Staged airbag deployments.

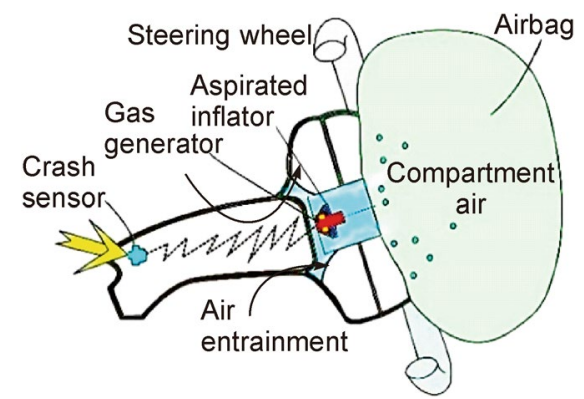

Fig. 3 Aspirated inflator operation based on the entrainment of ambient air using the supersonic pulse ejector 
The initial research objective for the aspirated inflation system was to inflate a 50-60 L driver's airbag within $30 \mathrm{~ms}$ with an aspiration ratio, $\boldsymbol{A} \geq 4$. The overall size should not exceed the length of $110 \mathrm{~mm}$ and a diameter of $120 \mathrm{~mm}$ to fit the size of the steering wheel column.

Reaching these goals results in inevitable conflicts between these requirements. Therefore, the solution is sought as an optimal compromise between separate engineering solutions satisfying the given requirements.

\section{Experiment Setup}

The investigations are implemented as matched numerical and experimental tasks formulated to satisfy the aspirated inflator engineering requirements. They start from the numerical evaluation of operation modes and corresponding design elements [10-12]. Unlike similar attempts based on the Coanda effect [14], the Prandtl-Meyer expansion fan is considered as a flow model to build a supersonic pulse ejector [12, 13, 15-17]. The primary (motive) supersonic jet flow, generated by a small gas generator, changes its direction over the blunt angle coupling between a nozzle and a mixing chamber, which causes a suction wave similar to that of Prandtl-Meyer flow. It initiates the ejection of the atmospheric air (secondary flow) into the mixing chamber and further into the cushion. A critical operation mode can arise at the velocity of the low-pressure secondary flow approaching the sound velocity. Then mixing of the two flows deteriorates, reducing the ejection efficiency. Thus, the investigation should focus on the proper high-pressure gas delivery into the mixing chamber by optimizing the system design.

The sought engineering solutions are essentially different from those characterizing stationary flows due to the different physical nature of processes taking place during pulse supersonic flow interaction with the entrained secondary flow [11, 12, 18-23].

\subsection{Research Strategy}

Figure 4 shows the numerically evaluated design of an axisymmetric supersonic pulse aspirator. Numerically, two temperature regimes were simulated. The hot gas case models the aspirated inflator operation with a gas generator producing the high-pressure jet, $T=1200^{\circ} \mathrm{K}$, with the required parameters. The cold gas case models the situation of compressed gas experiments jet, $T=300^{\circ} \mathrm{K}$.

Basic model elements of Fig. 4 that require special numerical and experimental design efforts are as follows:
- Linear and angular dimensions of the jet-forming ringtype nozzle and its coupling with a high-pressure zone of the pre-chamber;

- Type of coupling of the initial slit part with a diverging part of the nozzle;

- Pre-chamber and mixing chamber dimensions and shapes forming optimal flow patterns for the ambient air entrainment with a given flow rate and aspiration ratio;

- Location and the number of orifices between the prechamber and the gas generator;

- Matching of the inlet geometry of the aspirator at the atmospheric input pressure with the geometry and gasdynamic characteristics of its nozzle part;

- Coupling of an axisymmetric aspirator outlet with an airbag to provide a required excessive pressure in a given airbag.

Roughly evaluated design parameters are set to guide a further engineering design and experimental testing of the developed ejector model in a determined range of motive pressure, $P_{\mathrm{m}}$, values.

After finishing the engineering designs, several models are manufactured with the modified aspirator parts and their couplings, such as shapes and dimensions of its nozzle, prechamber, mixing chamber, etc. The experimental testing aims to validate and correct given numerical estimates in terms of the designed model operability in a given range of gas-dynamic parameters.

The measurement program comprises three separate tasks for each model design and a range of motive pressures:

- Measurement of pressure fields in the aspirator system;

- Measurement of entrainment/aspiration ratio;

- High-speed video-recording of the airbag inflation process.

Special attention is paid to determining optimal values of operational (motive) pressure for the given aspirator geometry. Patterns of pressure fields measured using distributed

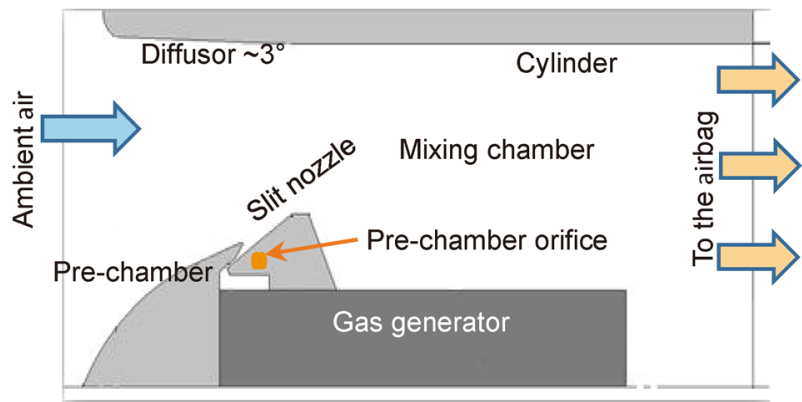

Fig. 4 Numerically evaluated aspirator design with recommended linear and angular dimensions 
pressure probes enable to determine a dominant jet location within the aspirator housing (mixing chamber) and to recommend optimal sets of parameters. Stable behavior of the supersonic jet within 30-40 ms implies a stable entrainment/ aspiration process which is not essentially deteriorated with flow separation or relocations of the initiating jet. Conclusions on the aspirator operability and its design recommendations are substantiated with measured aspiration ratios and the video-recorded airbag inflation process.

\subsection{Experiment Arrangement}

A dedicated compressed-gas experimental facility was designed and fabricated to test developed ejector systems $[18,24]$. It includes high-pressure tanks (25 MPa), reduction valve, receiver (15 MPa), pressure gauges, a high-speed valve, high- and low-pressure compressors, a data acquisition and processing system, and a high-speed camera.

The high-speed valve UHSV-1 is the key element of the facility for testing airbag inflation using aspirated inflators. It is specially designed [25] to mimic the supersonic jet generation in the automotive inflation system using the pyrocartridge. The time from the valve powering (control signal) to the start of its opening is $12 \mathrm{~ms}$. Figure 5 illustrates it for the input pressure $P_{0}=6.15 \mathrm{MPa}$.

Detailed technical characteristics of the valve are given in Table 1.

Thus, the short opening and closing times of the valve form a jet flow from the aspirator nozzle within $20-40 \mathrm{~ms}$ that initiates the ambient air entrainment. Under these conditions, the "cold gas" experiments in the pneumatic facility can be considered identical to the "hot gas" testing. Consequently, the results obtained numerically for both cases can be analyzed and compared with the measurement results. Besides, it can give a clue to predict hot-gas experimental results using cold-gas data taking into account their different gas-dynamic parameters, e.g., essentially different volume flow rates in the two cases.
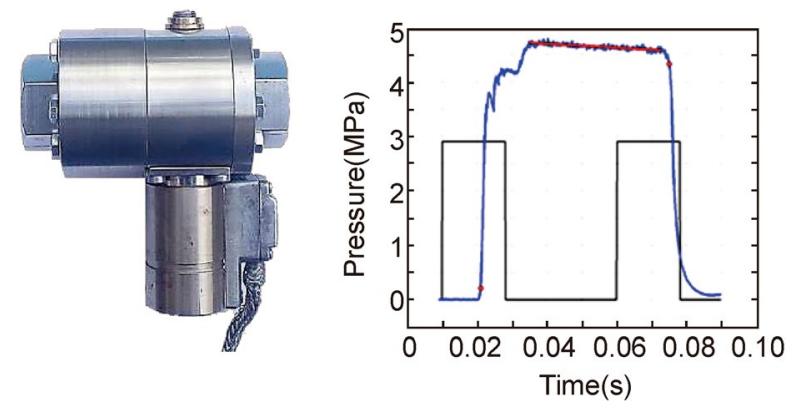

Fig. 5 High-speed valve (left) and its response (right, red curve) to the open-close control signals (black curve)

\section{Test Models and Arrangement of Measurements}

The data acquisition, processing, and control system is developed to launch and control the whole measurement process. The system is integrated into the measurement complex of the Advanced AIR laboratory [13, 18, 25-27]. The PCI-1710 multifunctional data acquisition board generates control signals and measures up to 15 signals from the pressure probes via 15 analog input channels.

The measurement complex can be managed both manually and remotely. LabView-specific software used to measure analog signals is developed and regularly updated following hardware modifications depending on experimental tasks. The MATLAB software package is used for data postprocessing and presentation of measured values. Thus, the designed measurement complex provides:

- Remote control;

- Remote measurement up to 100,000 sampling rate per second;

- Optical isolation of control circuits;

- Easy-to-access input-output connectors.

As a result, the operator can see the obtained real-time data in the flow parameter units and preliminarily analyze them. It enables adjustments of experimental conditions during testing and effective planning of further tasks.

\subsection{Aspirated Inflator Models}

According to the basic numerical recommendations (according to Fig. 4) and engineering designs, several inflator models were designed to enable some modifications during testing [10-12, 18, 24]. Their first set is characterized as inflators with an external slit [18] where the circumferential nozzle defines the overall size of an aspirator normally to its axis Fig. 6a. The second type combines aspirators with an internal ejecting slit/nozzle Fig. 6b, c and the overall unit dimensions are those of a mixing chamber

Table 1 High-speed valve technical parameters

\begin{tabular}{ll}
\hline Opening time & $2-4 \mathrm{~ms}, \max$ \\
\hline Closing time & $3-5 \mathrm{~ms}, \max$ \\
Pressure range & $0.1 \sim 6.5 \mathrm{MPa}$ \\
Temperature range & $+5 \sim+65^{\circ} \mathrm{C}$ \\
Voltage & $80 \pm 6 \mathrm{~V}$ \\
Material & Steel $14 \times 17 \mathrm{H} 2$ \\
Weight & $3 \mathrm{~kg}$ \\
Dimensions & $79 \mathrm{~mm} \times 125 \mathrm{~mm} \times 141 \mathrm{~mm}$ \\
\hline
\end{tabular}


where the interaction of the high-speed jet and the entrained airflow takes place. The sharp bend edge responsible for Prandtl-Meyer conditions is given by the coupling of adjacent A and B surfaces of two metal disks \#1 and \#2.

Variable parameters during testing are motive pressure $P_{\mathrm{m}}$ in the pre-chamber and design geometry. For example, the modifying design elements in Fig. 6b, c are:

- Angular and linear sizes of nozzle-forming surfaces (see, e.g., Figs. 6c, 7);

- A nozzle slit width can be changed in the course of testing within $0.3-0.5 \mathrm{~mm}$ with a step of $0.05 \mathrm{~mm}$;

- The shape of the internal geometry housing (mixing chamber): the cylinder conjugated with a diffusor like in Fig. 4 or any other combinations.

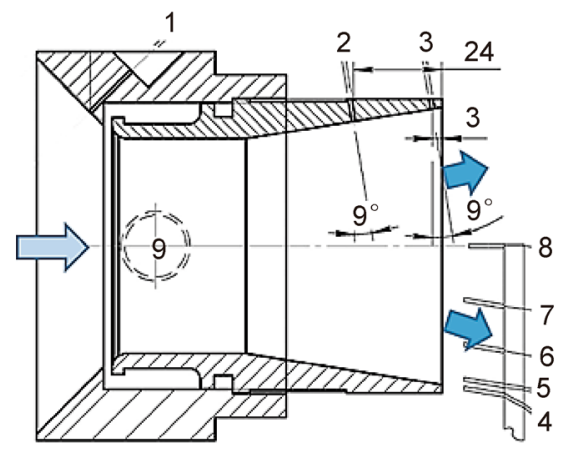

(a)

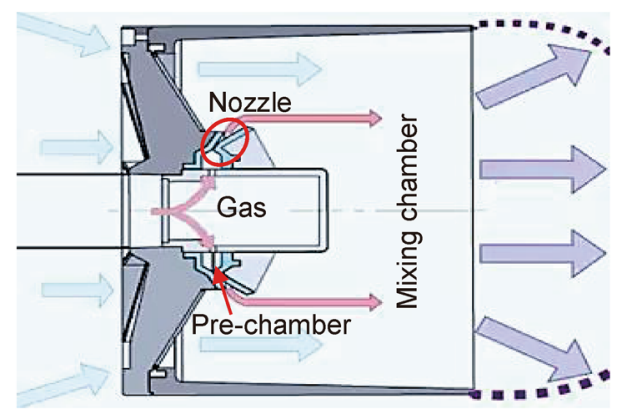

(b)

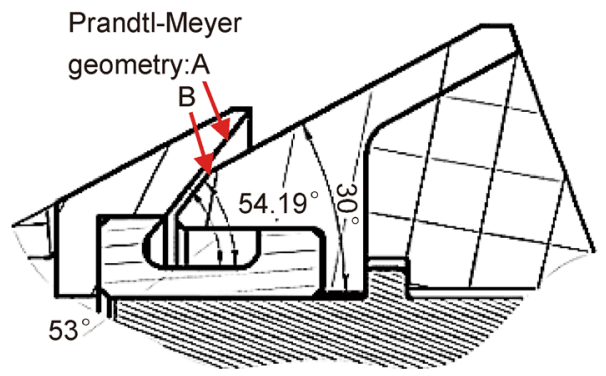

(c)

Fig. 6 Two basic designs of the aspirated inflator realizing the Prandtl-Meyer conditions: a external nozzle with locations of pressure probes, 1-8; $\mathbf{b}$, $\mathbf{c}$ internal nozzle with shown jet-forming elements
Figure 7 shows a sketch of the aspirated inflator with the internal nozzle used in the numerical simulation. The calculations were made for various locations of nozzle singular points $\mathrm{A}, \mathrm{B}, \mathrm{C}, \mathrm{D}$, thus obtaining different linear dimensions including the slit-nozzle width $h$ and varying angles $\alpha, \beta, \gamma$.

Figure 8 shows two basic fabricated models of the aspirated inflator with the \#1 external nozzle and with the \#2 internal nozzle. Both are characterized by sets of design parameters varying in experiments.

\subsection{Measurement of Pressure Fields in the Aspirated Inflator}

The assembled inflators mounted in the compressed-air facility are shown in Figs. 9 and 10 together with the distributed system of pressure probes. A pressure probe comb measuring the dynamic pressure along the inflator radius and pressure probes inbuilt along the mixing chamber generatrix display the pressure field within the inflator. Also, it enables to follow the jet location and its behavior depending on the operating pressure.

The \#1 pressure probe (Fig. 10) measures the pressure in the pre-chamber where the pressurized gas enters from the high-pressure source (compressed gas facility or the pyro-cartridge). Full pressure at the exit from the inflator is measured in a radial direction using the pressure-probe rake of \#2-10 probes marked red in Fig. 10; the static pressure along the wall of a mixing chamber is measured with the 5 embedded probes \#11-15 marked green.

Pressure field measurements are supplemented with the high-speed video-recordings of the airbag deployment process and with measurements of aspirated ratio for various versions of the inflator design features in combination with the high-pressure values.

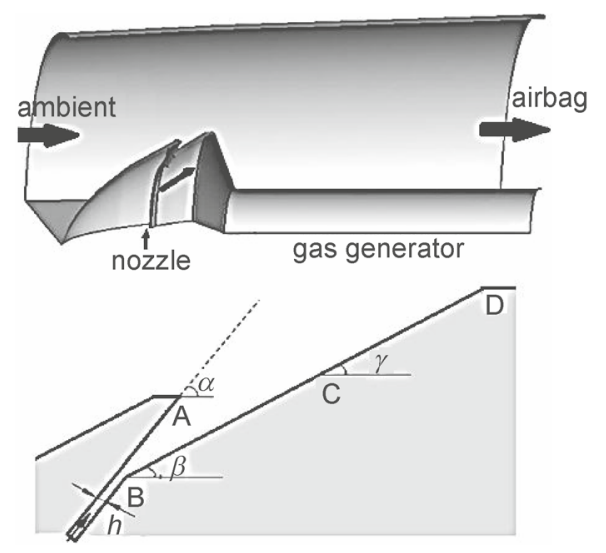

Fig. 7 Variable parameters of the nozzle geometry 

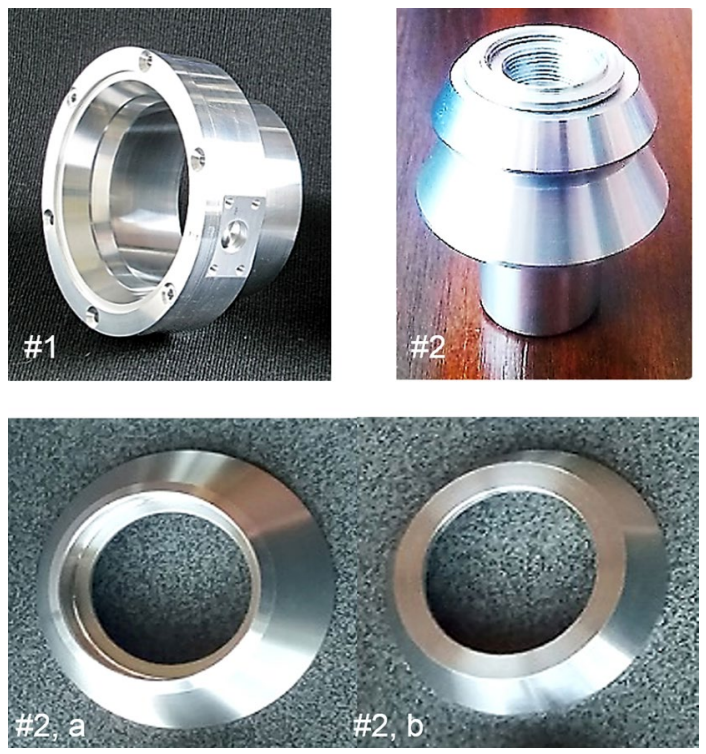

Fig. 8 The fabricated aspirated inflator test models with external nozzle \#1 and with the internal nozzle \#2; jet forming disks \#2,a and \#2,b for the assembly \#2

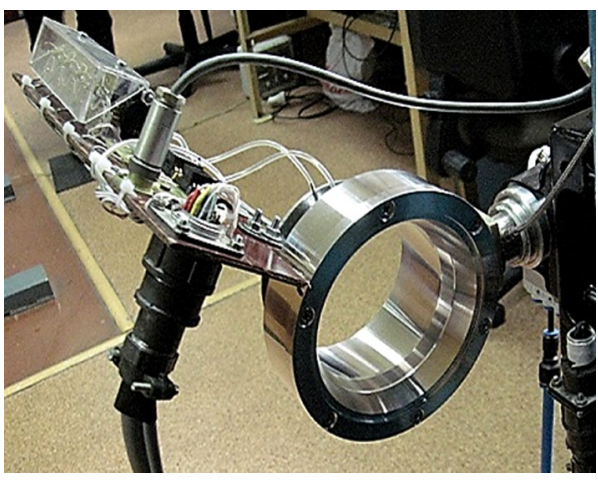

Fig. 9 The external-slit aspirated inflator mounted in the compressedair facility

\section{Results and Discussion of Measured Pressure Distributions}

\subsection{Pressure Range}

The basic goal of pressure measurements is to determine conditions that provide the stable behavior of the supersonic jet and, consequently, the stable entrainment/aspiration process. Flow separation effects or just the unstable location of the jet in the mixing chamber can deteriorate this process. To mitigate the consequences of the improper flow behavior $[12,13,18]$, experimental efforts were

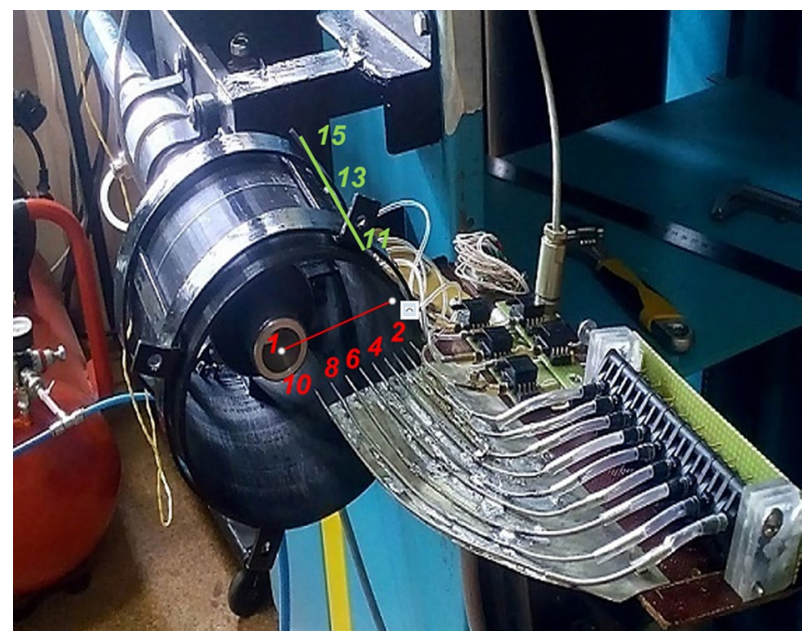

Fig. 10 The internal-slit aspirated inflator in the compressed-air facility with the pressure probe system

focused on finding optimal ranges of parameters such as operational (motive) pressure, $P_{\mathrm{m}}$, for the given aspirator geometry.

The pressure measurements discussed below refer to the aspirator of Fig. 8, \#2 with the internal nozzle. Fifteen patterns of measured pressure, $P(t)$, enable to reconstruct the flow structure, to determine a dominant jet position within the aspirator housing, and to recommend optimal combinations of operation parameters.

Figure 11 shows such typical variations of dynamic pressure with time using one of the internal-slit aspirator models.

These patterns serve as a basis for analyzing the jet behavior during inflation time. The initiating pulse from a high-speed valve is marked with a black line; red lines correspond to averaged (de-noised) values of pressure measured by the probes. Figure 11 shows that meaningful signals are registered by near-wall \#2 and \#3 probes, thus indicating the jet location at the aspirator housing wall.

Correspondingly, Fig. 12 shows the reconstructed flow field with the jet propagated to the outer edge of the mixing chamber.

Decreasing the operating pressure by $0.3-0.5 \mathrm{MPa}$, one can observe the jet relocation toward the aspirator axis. As the high $P_{0}$ pressure is reduced to $4.8 \mathrm{MPa}$ (Fig. 13), the jet propagates almost parallel to the aspirator axis (Fig. 14). Its behavior becomes much more stable that is characterized by the meaningful signals of all the full-pressure probes \#2-10.

Compared to Fig. 11 case, the jet stabilization also results in the formation of the stable suction pressure zone along the mixing chamber wall (Fig. 13, the right column of \#11-15 probes).

On the one hand, the obtained results can feature some instability of jet behavior; on the other hand, the system 

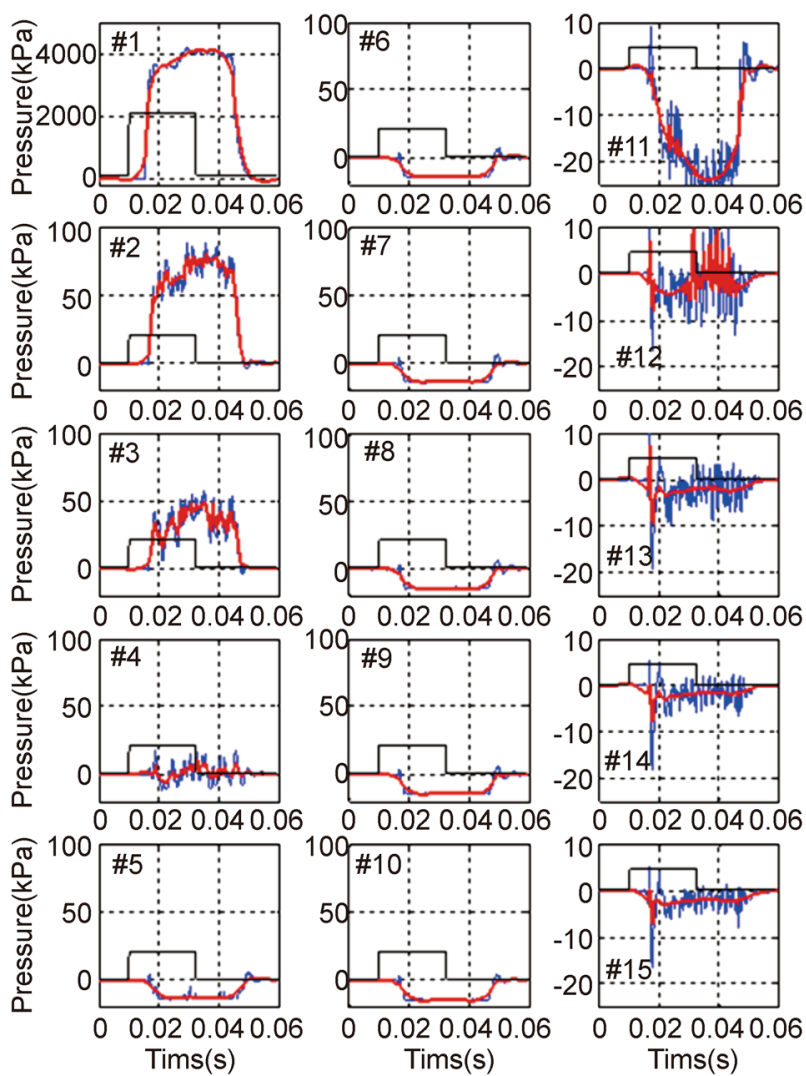

Fig. 11 Pressure measured in 15 probe locations for the aspirator model of Figs. $6 \mathrm{~b}, \mathrm{c}$ and 8, \#2: operating high pressure, $P_{0}=5.1 \mathrm{MPa}$; slit width, $h=0.4 \mathrm{~mm}$ (Fig. 10)

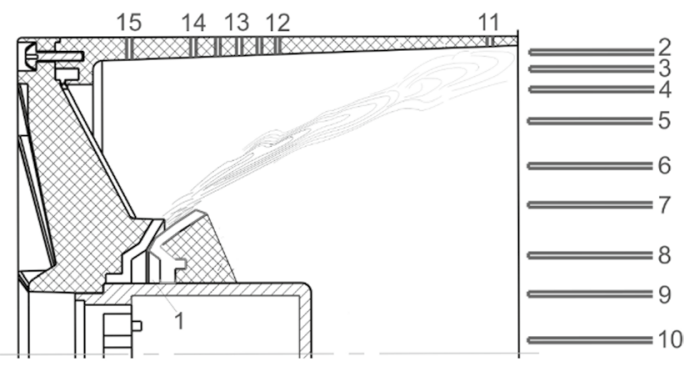

Fig. 12 Supersonic jet propagating to the aspirator wall for conditions of Fig. 11

sensitivity to variations of motive pressure imposes specific requirements to the gas generator characteristics.

Preliminary numerical estimation showed that the operation of the considered aspirator design is satisfactory under the condition of the high pressure varying by no more than within $25 \%$ of its optimal calculated value, i.e., $4.2 \mathrm{MPa} \leq P_{0} \leq 5.4 \mathrm{MPa}$.
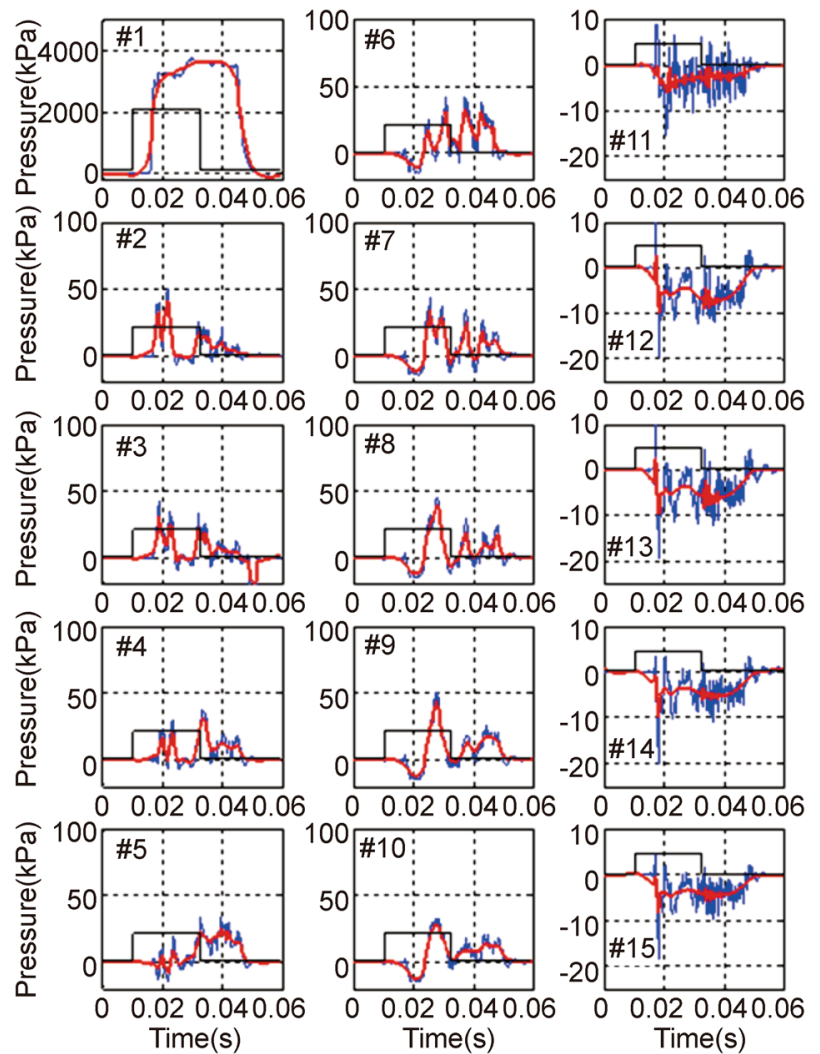

Fig. 13 Pressure field in the aspirator model of Fig. 6b, c: $P_{0}=4.8 \mathrm{MPa}$; slit width, $h=0.4 \mathrm{~mm}$

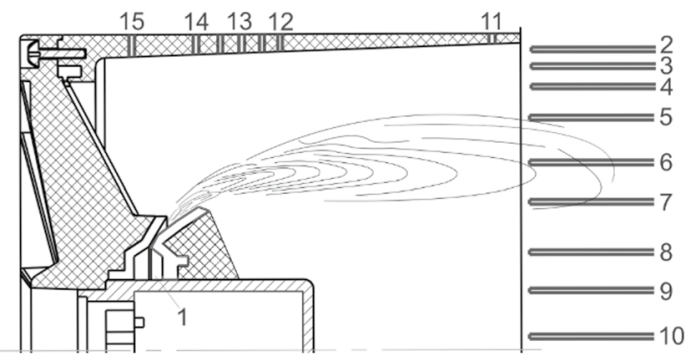

Fig. 14 Supersonic jet propagation along the aspirator axis for conditions of Fig. 13

\subsection{Aspiration Ratio}

In the optimal case of Figs. 13, 14, the static pressure distribution registered by the \# 11-15 probes clearly shows the suction area during the whole period of inflation, $t=10-50 \mathrm{~ms}$ (Fig. 13, right column). This vacuum pressure decreases from $\sim 5$ to $\sim 3 \mathrm{kPa}$ with growing high input pressure that, in turn, results in the falling ambient air entrainment. Indeed, this tendency is well seen both from 
the results of numerical simulation and from direct measurements of the aspiration ratio depending on the motive pressure, i.e., the pressure before the nozzle measured by the \#1 probe in the pre-chamber.

Figure 15 shows the calculated and measured aspiration ratio values falling with growing motive pressure for the internal-nozzle aspirator design of Fig. 10. The best air entrainment is found for the pressure 0.1-0.2 MPa less than the pressure indicative of the jet displacement toward the aspirator wall, i.e., for the jet propagating almost parallel to the aspirator axis where it demonstrates its stable behavior.

Experimental data are in good agreement with those predicted numerically for the relevant "cold gas" case of $300 \mathrm{~K}$. Since the experimental aspiration ratio curve goes above the numerical one for $300 \mathrm{~K}$, one can suppose a similar trend for the $1200 \mathrm{~K}$ "hot gas" case. That is, in practice, the volume aspiration ratio is expected to roughly double the numerically predicted values (orange) for the aspirator driven by a pyro-cartridge.

The given engineering requirements can be satisfied only under conditions of certain compromises between the choice of aspirator geometry and the pressure range of its operation. Besides, there are ways to improve the aspirator efficiency. For example, the red curve in Fig. 15 shows higher aspiration ratio values for the slightly optimized design [15-17] that enhanced the air entrainment.

\subsection{Airbag Inflation}

The airbag inflation process was video-recorded for both cold and hot gas cases using the developed aspirated inflators. The high-speed recording technique made it possible to analyze the process in detail, comparing the results obtained for different sets of parameters. As an example, Fig. 16 shows consecutive stages of airbag deployment using the

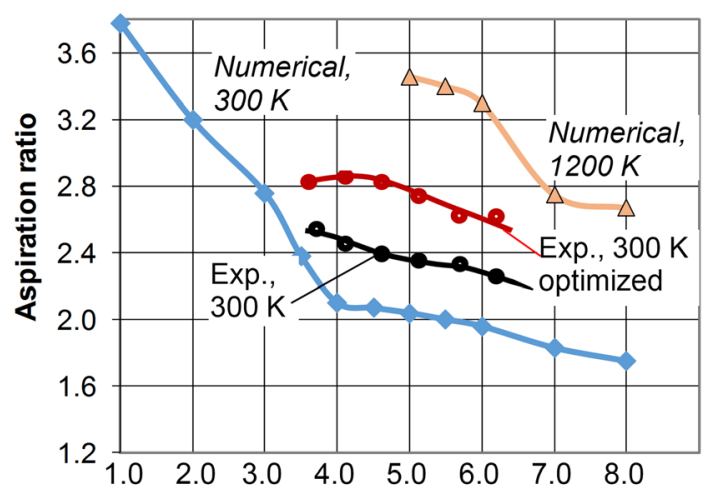

Motive pressure, $\mathrm{MPa}$

Fig. 15 Aspiration ratio versus high input pressure, $A\left(P_{0}\right)$
\#1 inflator of Figs. 8 and 9 with the external nozzle. The inflation process in the compressed-air facility, $T=300 \mathrm{~K}$ case (Fig. 16, top), was recorded for an optimized couple of basic variables, nozzle width and motivated high-pressure.

Similar conditions for the case $T=1200 \mathrm{~K}$ were created in the facility where the pressurized supersonic jet flow was generated using a pyro-cartridge (Fig. 16, bottom, and Fig. 17). High-temperature gas in the second case provides a visually bigger volume and fullness of a cushion, and this appears to be sufficient to mitigate crash consequences as it is shown in Fig. 17. It is in good agreement with the numerical prediction of the significantly greater inflated gas volume, if its temperature is much higher than that in the pneumatic experiments.

\section{Conclusions}

The aspirated inflator applicable to airbags of conventional and autonomous vehicles is developed due to matching numerical and experimental efforts. Two basic models and their several modifications are built and tested: with an external circumferential slit nozzle and with an internal nozzle placed in the mixing chamber. Both aim at the entrainment of the $3 / 4$ of the airbag air volume from the vehicle compartment that requires $\sim 1 / 3$ of the conventional propellant amount. It also reduces a traumatic airbag impact on the vehicle occupants due to the pressure and temperature control in the compartment and stopped airbag deployment on contact with an occupant. In turn, the latter eliminates the need for occupant weight and out-of-position sensors.
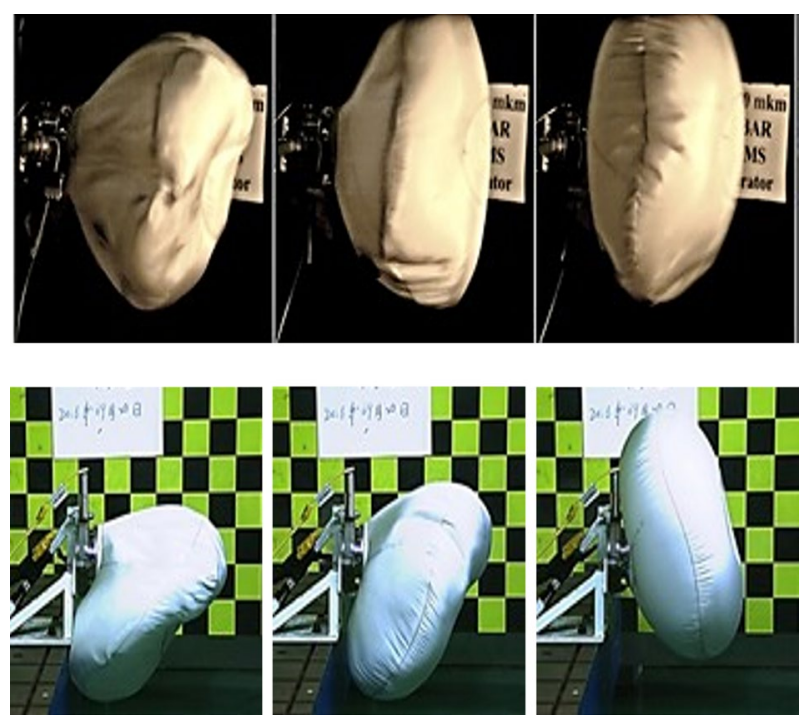

Fig. 16 Time sequences of the airbag deployment using the aspirated inflator with an external slit-nozzle $0.25 \mathrm{~mm}$ wide: top-pneumatic facility, cold gas, $P_{0}=3.6 \mathrm{MPa}$; bottom-pyro-cartridge, hot gas 

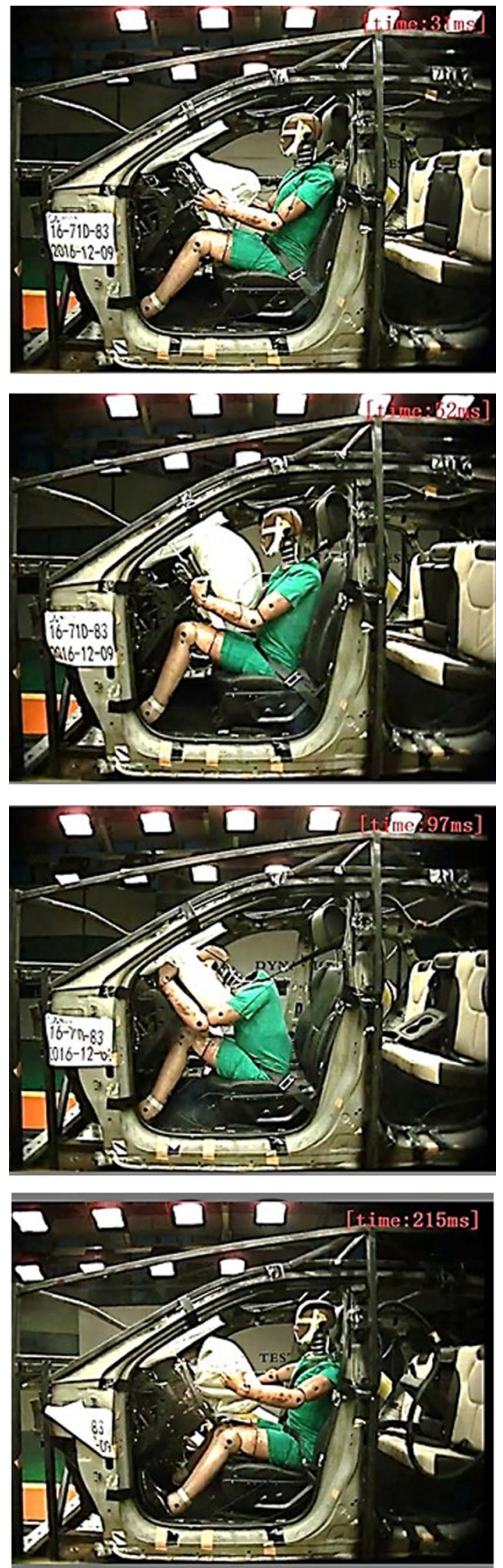

4Fig. 17 Sled test with the airbag deployment using the external-slit aspirated inflator

(1) The Prandtl-Meyer expansion fan problem is taken as a starting point for the numerical modeling of inflator design. It enabled to obtain guiding linear and angular dimensions of the inflator nozzle and other parts. The slit nozzle shapes the supersonic pulse jet responsible for the atmospheric air suction into the mixing chamber and the cushion. Thus, the arrangement of the proper pressure distribution within the inflator is a focal issue of this research.

(2) A group of fabricated experimental models is tested for a driver airbag to determine their operational effectiveness in a range of high motive pressure values. Experiments are carried out using the pneumatic facility equipped with a high-speed valve mimicking the inflator operation with the pyrotechnic gas generator. Multivariate measurements of pressure fields, high-speed video-recording of the airbag inflation process, and evaluation of aspiration (entrainment) ratio characterize the aspirated inflator operability.

(3) Obtained numerical and experimental results show ranges of parameters characterizing the device geometry and operation. They are high motive pressure combined with the slit nozzle shape and width, size of the pre-chamber, shape and width of the mixing chamber, as well as couplings of separate elements. As a result, optimal sets of parameters are recommended. Measured aspiration ratio values and videorecorded airbag inflation verify these recommendations.

Thus, theory and computation-based findings combined with their empirical validation and experimental design improvements prove the developed concept applicability to the airbag aspirated inflators. With the aim of further practical applications, the research should be continued in two primary directions: (1) aerodynamic optimization of the inflator design, (2) development of the numerical basis and experimental design/testing of plane airbag inflation systems for bigger airbags.

Acknowledgements The authors acknowledge with thankfulness the financial support and fruitful collaboration with the Shanghai East Joy Long Motor Airbag Co Ltd.

\section{Declarations}

Conflict of interest On behalf of all the authors, the corresponding author states that there is no conflict of interest.

Open Access This article is licensed under a Creative Commons Attribution 4.0 International License, which permits use, sharing, adaptation, distribution and reproduction in any medium or format, as long as you give appropriate credit to the original author(s) and the source, provide a link to the Creative Commons licence, and indicate if changes were made. The images or other third party material in this article are included in the article's Creative Commons licence, unless indicated otherwise in a credit line to the material. If material is not included in 
the article's Creative Commons licence and your intended use is not permitted by statutory regulation or exceeds the permitted use, you will need to obtain permission directly from the copyright holder. To view a copy of this licence, visit http://creativecommons.org/licenses/by/4.0/.

\section{References}

1. Jiao, N., Ghaffarzadeh, K., Jiang, L.Y.: Autonomous cars and robotaxis 2020-2040: players, technologies, and market forecast. IDTechEx Research Reports, 05 April (2020). https://www.idtec hex.com/en/research-report/autonomous-cars-and-robotaxis2020-2040-players-technologies-and-market-forecast/701

2. FABULOS (Future Automated Bus Urban Level Operation Systems) project: https://fabulos.eu/fabulos-project/

3. Global Times: China sets eyes on autonomous vehicle growth, leader in global standards. Published, 19 May (2020). https:// www.globaltimes.cn/content/1188795.shtml

4. NHTSA Issues first-ever proposal to modernize occupant protection safety standards for vehicles without manual controls, 17 March (2020). https://www.nhtsa.gov/press-releases/adapt-safetyrequirements-ads-vehicles-without-manual-controls

5. Regulation of the European parliament and of the council: Concerning type-approval requirements for the general safety of motor vehicles. Commission of the European Communities, Brussels, pp.55, 18 October (2019)

6. Holtz, J., Müller, G., Johannsen, H., et al.: Hearing loss analysis in full-scale accident reconstruction. In: Proceedings 26th Internationl Technical Conference on the Enhanced Safety of Vehicles. Eindhoven, Netherlands, Paper \# 19-0028, 10-13 June (2019)

7. ZF Develops Seat-Integrated Advanced Center Airbag Concept: ZF press release, 2017-Jun-06 https://press.zf.com/press/en/relea ses/release_2773.html

8. Autoliv Future Technologies, life cell airbag: https://www.autol iv.com/products/passive-safety/future-technologies

9. Schöneburg, R., Hart, M., Feese, J., Mücke, S., et al.: Experimental safety vehicle meets automated driving mode. In: Proceedings 26th International Technical Conference on the Enhanced Safety of Vehicles. Eindhoven, Netherlands, Paper \# 19-0042, 10-13 June (2019)

10. Yurchenko, N.: Supersonic ejectors for novel airbag inflation systems. Academia Letters - Open Access, Article 796 (2021), https://doi.org/10.20935/AL796

11. Breed, D., Voropaiev, G., Yurchenko, N.: Airbag inflators including aspirators. Internat patent application number PCT/ US2018/013088, Int. Publication \# WO 2018/132420 A1, published 19 July (2018)

12. Breed, D., Zhang, S., Yurchenko, N., et al.: Toward autonomous vehicles: basic aerodynamic background for novel airbag inflation system. In: Proceedings of the 14th International Symposium on Sophisticated Car Safety Systems. Mannheim, Germany, pp.17 26-28 Nov (2018)

13. Kuzmenko, K.M., Yurchenko, N.F., Vynogradskyy P.M., et al.: Operational characteristics of a supersonic ejector depending on pressure. In: Proceedings of the International Conference on Hydro-aerodynamics in the Engineering Practice. National Technical University of Ukraine "I. Sikorsky Kyiv Polytechnic Institute", Kyiv, Ukraine (2018)
14. Wang, C.J., Sung, K.: The numerical analysis and design of the aspirated inflator on backside airbag, pp. 1-9 (2009). https://doi. org/10.29770/JTCMT.200903.0001

15. Yurchenko, N.F.: Energy-efficient flow control around blunt bodies. Int. J. Adv. Aircraft Spacecraft Sci. 1(1), 15-25 (2013). https://doi.org/10.12989/aas.2013.1.1.000

16. Yurchenko, N.: Smart flow control based on generated spanwise flow regularity. Lectures, Dep. of aerospace engineering \& Dep. of chemical/applied sciences, Delft University of Technology, Netherlands, 4 Dec (2018)

17. Breed, D., Yurchenko, N., Vynogradskyy, P., Kuzmenko, K., et al.: The analysis and experimental development of aspirated airbags for conventional and autonomous vehicles. In: Proceedings of the 26th International Technical Conference on the Enhanced Safety of Vehicles. Eindhoven, Netherlands, Paper \# 19-0025, 122-131, 10-13 June (2019)

18. Breed, D., Zhang, S., Yurchenko, N., et al.: Development of an aspirated inflator for preventing out-of-position occupant injuries. In: Proceedings of the 14th International Symposium on Sophisticated Car Safety Systems, Mannheim, Germany, 26-28 Nov (2018)

19. Addy, A.L: The analysis of supersonic ejector systems. AGARDograph No 163 on Supersonic Ejectors, Ed. J.J.Ginoux, pp. 31-103 (1972)

20. Al-Nuaimi, A., Worall, M., Rifat, S.: Preliminary study of effects of primary and back pressure on performance of supersonic ejector driven by compressed air. In: 17th International Conference on Sustainable Energy Technologies, Wuhan, China 21-23rd August (2018)

21. Lamberts, O., Chatelain, P., Bartosiewicz, P.: Experimental and numerical analysis of the flow within the supersonic ejector. In: Proceedings of ICHMT International Symposium on Advances in Computational Heat Transfer, Napoli, Italy, 28 May-1 June (2017)

22. Kurochkin, A.I., Imaev, S.Z.: The optimization of ejector systems for the gas industry. Oil \& Gas Refining, \#6 (in Russian) (2017)

23. Im, K.S., Zhang, Z.C., Grant, O.: Airbag inflator models in LSDYNA. In: 14th International LS-DYNA Conference, Session: Occupant Safety, pp. 1-1-1-4, 12-14 June (2016)

24. Kuzmenko, K., Yurchenko, N., Vynogradskyy, P., et al.: Optimization of ejector design and operation. EPJ Web of Conferences, vol. 114, Paper No. 02063, (2016). https://doi.org/10.1051/epjco nf/201611402063

25. Kysil, V.L., Kuzmenko, K.M.: Universal high-speed pneumatic valve UHSV16-1. In: Proceedings of the International Conference on Hydro-aerodynamics in the Engineering Practice, National Technical University of Ukraine "I. Sikorsky Kyiv Polytechnic Institute", Kyiv, Ukraine (in Ukrainian) 7-10 Oct. (2019)

26. Vynogradskyy, P., Yurchenko, N., Pavlovsky, R., et al.: Aerodynamic facility with MW-systems for flow control based on localized plasma generation. AIAA Paper-2008-3939. Semantic Scholar. https://doi.org/10.2514/6.2008-3939 Corpus ID: 113328934 (2008)

27. Yurchenko, N., Vynogradskyy, P., Paramonov, Y., et al.: Aerodynamic complex for interdisciplinary research with an integrated system for data acquisition and processing. In: Proceedings of the World Congress, Aviation in the XXI-st Century. National Aviation University, Kyiv, Ukraine (2012) (in Russian) 